\title{
Variable stability system control law development for in-flight simulation of pitch/roll/yaw rate and normal load
}

\section{Joon Soo Ko*}

School of Aerospace and Mechanical Engineering, Korea Aerospace University, Goyang-si(412-791), Republic of Korea

\section{Sungsu Park**}

Department of Aerospace Engineering, Sejong University, Seoul, Republic of Korea

\begin{abstract}
This paper describes the development of variable stability system (VSS) control laws for the KFA-i to simulate the dynamics of KFA-m aircraft. The KFA-i is a single engine, Class IV aircraft and was selected as an in-flight simulator (IFS) aircraft, whereas the KFA-m is a simulated aircraft that is based on the F-16 aircraft. A 6-DoF math model of KFA-i aircraft was developed, linearized, and separated into longitudinal and lateral motion for VSS control law synthesis. The KFA-i aircraft has five primary control surfaces: two flaperons, two all movable horizontal tails, and one rudder. Flaperons are used for load control, the horizontal tails are used for pitch and roll rate control, and the rudder is used for yaw rate control. The developed VSS control law can simulate four parameters of the KFA-m aircraft simultaneously, such as pitch, roll, yaw rates, and load. The simulation results show that KFA-i follows the responses of KFA-m with high accuracy.
\end{abstract}

Key words: variable stability system, in-flight simulation, model-following, fly-by-wire, fader

\section{Introduction}

Modern fighter aircraft employs full-authority digital flyby-wire flight control system and the proper evaluation of sophisticated flight control system is important. An in-flight simulation environment can be used for handling quality evaluation and for flying the newly developed control laws [1]. For in-flight simulation, a well-proven aircraft is used as an inflight simulator (IFS) aircraft with a variable stability system (VSS) embedded on it. VSS can alter the static and dynamic characteristics of an aircraft over a wide range and thus can provide in-flight simulation capability for the aircraft [2]. By changing an IFS aircraft's stability and flying characteristics to match those of another aircraft - i.e. the simulated aircraft - the simulated aircraft's flight control law is verified and validated $[3,4]$. The principal applications of IFS are handling quality investigation, pilot-induced oscillation (PIO) evaluation, and the verification of new control methods without extensive modification of the IFS aircraft.

This paper presents a model-following VSS control law design and validation for in-flight simulation. The KFA-i was selected as the IFS aircraft; it is a single-engine and Class IV aircraft. The KFA-m was selected as the simulated aircraft, which is based on the F-16. A 6-DoF math model of the KFA-i aircraft was developed, linearized, and separated into longitudinal and lateral motion for VSS control law synthesis. The designed control law has been analyzed and implemented for simulating 6-DoF math model of the KFA-m.

On the basis of the KFA-i aircraft with static type of autopilot for stability provision, VSS control laws for simulation of the KFA-m dynamics (pitch, roll, and yaw rates, and normal load factor) was developed using a model-following technique for the flight condition points, named $\mathrm{HH}, \mathrm{LH}$, and LL. Investigated flight conditions are shown in Table 1.
This is an Open Access article distributed under the terms of the Creative Commons Attribution Non-Commercial License (http://creativecommons.org/licenses/by$\mathrm{nc} / 3.0 /$ which permits unrestricted non-commercial use, distribution, and reproduction in any medium, provided the original work is properly cited. (cc) Associate Professor, Corresponding author: jsko@kau.ac.kr
** Professor, sungsu@sejong.ac.kr 
Joon Soo Ko Variable stability system control law development for in-flight simulation of pitch/roll/yaw rate and normal load

Table 1. Flight conditions for IFS

\begin{tabular}{|c|c|c|c|}
\hline Flight Condition & LH & LL & HH \\
\hline \multirow{2}{*}{ KFA-i } & $\mathrm{H}=10 \mathrm{kft}$ & $\mathrm{H}=40 \mathrm{kft}$ & $\mathrm{H}=40 \mathrm{kft}$ \\
& $\mathrm{M}=0.8$ & $\mathrm{M}=0.9$ & $\mathrm{M}=0.95$ \\
\hline \multirow{2}{*}{ KFA-m } & $\mathrm{H}=5 \mathrm{kft}$ & $\mathrm{H}=5 \mathrm{kft}$ & $\mathrm{H}=40 \mathrm{kft}$ \\
& $\mathrm{M}=0.74$ & $\mathrm{M}=0.33$ & $\mathrm{M}=0.95$ \\
\hline
\end{tabular}

\section{Design of Model-following Control}

There are two methods available to achieve in-flight simulation capability: response-feedback and modelfollowing techniques. A response-feedback technique modifies the dynamics of the aircraft through the response feedback loops and gains, but it is limited in its capacity to model dissimilar aircraft dynamics. A model-following control system forces the in-flight simulation aircraft to respond according to a pre-programmed aircraft dynamic model, and it allows high accuracy of simulation over widely dissimilar aircraft dynamics [5]. Most advanced in-flight simulations are, therefore, based on model-following control systems $[6,7]$.

Given the equation for the motion of an in-flight simulation aircraft,

$$
\dot{\mathbf{x}}_{p}=\mathbf{A}_{p} \mathbf{x}_{p}+\mathbf{B}_{p} \mathbf{u}_{p}
$$

and the equation of motion of a simulated aircraft,

$$
\dot{\mathbf{x}}_{m}=\mathbf{A}_{m} \mathbf{x}_{m}+\mathbf{B}_{m} \mathbf{u}_{m}
$$

the model-following control algorithms are formalized as follows: determine the control law $\mathbf{u}_{p}$ such that the exact model-following is achieved,

$$
\mathbf{x}_{p}=\mathbf{x}_{m} \text { and } \dot{\mathbf{x}}_{p}=\dot{\mathbf{x}}_{m}
$$

where $\mathbf{A}_{p}$ and $\mathbf{A}_{m}$ are state matrices, $\mathbf{B}_{p}$ and $\mathbf{B}_{m}$ are control matrices, $\mathbf{x}_{p}$ and $\mathbf{x}_{m}$ are the $\mathrm{n}$ state variables, $\mathbf{u}_{p}$ and $\mathbf{u}_{m}$ are the $\mathrm{m}$ control variables, and the subscripts $\mathrm{p}$ and $\mathrm{m}$ denote the corresponding plant (IFS aircraft) and model (simulated

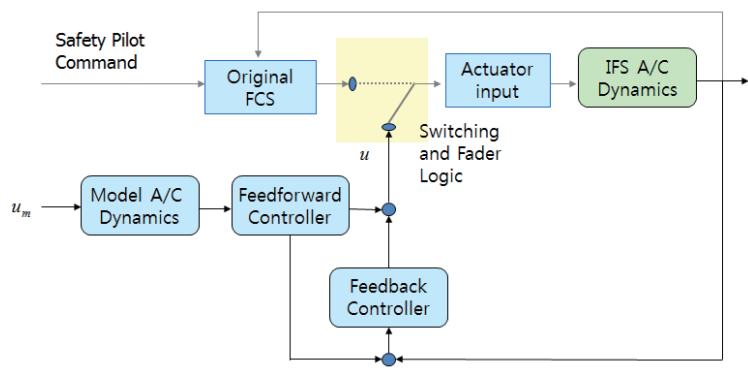

Fig. 1. Model-following variable stability control system aircraft) states and parameters, respectively.

The proposed control system in this paper for achieving the goal of in-flight simulation consists of feed-forward and feedback control laws, the aircraft dynamic model to be simulated, baseline flight control system, and switching and fader logics. Fig. 1 shows the architecture of this control system.

\subsection{Feed-forward Control}

Equation 3 can be accomplished if the control law $\mathbf{u}_{p}$ is chosen as follows:

$$
\mathbf{u}_{p}=\mathbf{B}_{p}^{+}\left(\dot{\mathbf{x}}_{m}-\mathbf{A}_{p} \mathbf{x}_{m}\right)
$$

where, $\mathbf{B}_{p}^{+}=\left(\mathbf{B}_{p}^{T} \mathbf{B}_{p}\right)^{-1} \mathbf{B}_{p}^{T}$

Conceptually, this controller cancels the bare dynamics of the IFS aircraft and elicits the model aircraft dynamics as the model would do to a given model input $\mathbf{u}_{m}$.

The VSS control law objective in this paper is to simulate four parameters - pitch, roll, yaw rates, and load - of the KFA-m aircraft simultaneously. The KFA-i aircraft has five primary control surfaces: two flaperons, two all movable horizontal tails, and one rudder. Flaperons are used for load control, the horizontal tails are used for pitch and roll rate control, and the rudder is used for yaw rate control.

For pitch rate simulation using horizontal tail symmetric (HTS), Equation 1 is rewritten in the following form for KFA-i pitch rate.

$$
\dot{q}=\bar{M}_{q} q+\bar{M}_{\alpha} \Delta \alpha+\bar{M}_{V} \Delta V+\bar{M}_{\theta} \Delta \theta+\bar{M}_{\delta_{H T S}} \delta_{H T S}+\bar{M}_{\delta_{T E F}} \delta_{T E F}+\bar{M}_{\delta_{R U D}} \delta_{R U D}
$$

where $q, \Delta \alpha, \Delta V, \Delta \theta$ are the pitch rate, angle of attack, forward velocity, and the pitch angle, respectively, and $\bar{M}_{q, \alpha, V, \theta}$ are pitching moment derivatives with respect to them. $\delta_{H T S}, \delta_{T E F}, \delta_{R U D}$ are HTS, TEF, and rudder deflections, respectively. Then, based on the model-following control in Equation 4, the HTS deflection is calculated as follows.

$$
\delta_{H T S}=\frac{\dot{q}_{m}-\left(\bar{M}_{q} q_{m}+\bar{M}_{\alpha} \Delta \alpha_{m}+\bar{M}_{V} \Delta V_{m}+\bar{M}_{\theta} \Delta \theta_{m}+\bar{M}_{\delta_{T E F}} \delta_{T E F}+\bar{M}_{\delta_{\text {EUD }}} \delta_{R U D}\right)}{\bar{M}_{\delta_{H T S}}}
$$

where $q_{m}$ is the pitch rate of KFA-m aircraft to be simulated 
by KFA-i. The states of the KFA-i aircraft in Equation 5 are replaced by the corresponding states of the KFA-m aircraft in Equation 6. In a similar way, for roll rate simulation using horizontal tail asymmetric (HTA), the following form of the roll rate equation is used.

$$
\dot{p}=\bar{L}_{\beta} \Delta \beta+\bar{L}_{p} p+\bar{L}_{r} r+\bar{L}_{\phi} \Delta \phi+\bar{L}_{\delta_{H T A}} \delta_{H T A}+\bar{L}_{\delta_{R U D}} \delta_{R U D}
$$

where $\Delta \beta, p, r, \Delta \phi$ are the sideslip angle, roll rate, yaw rate, and the roll angle, respectively, and $\bar{L}_{\beta, p, r, \phi}$ are rolling moment derivatives with respect to them. Then, based on the model following-control in Equation 4, the HTA deflection, $\delta_{H T A}$, is calculated as follows.

$$
\delta_{H T A}=\frac{\dot{p}_{m}-\left(\bar{L}_{\beta} \Delta \beta_{m}+\bar{L}_{p} p_{m}+\bar{L}_{r} r_{m}+\bar{L}_{\phi} \Delta \phi_{m}+\bar{L}_{\delta_{R U D}} \delta_{R U D}\right)}{\bar{L}_{\delta_{H T A}}}
$$

where $p_{m}$ is the roll rate of KFA-m aircraft to be simulated by KFA-i. Now, for yaw rate simulation using rudder, following form of the directional equation is used.

$$
\dot{r}=\bar{N}_{\beta} \Delta \beta+\bar{N}_{p} p+\bar{N}_{r} r+\bar{N}_{\phi} \Delta \phi+\bar{N}_{\delta_{H T A}} \delta_{H T A}+\bar{N}_{\delta_{R U D}} \delta_{R U D}
$$

where $\bar{N}_{\beta, p, r, \phi}$ are yawing moment derivatives with respect to $\Delta \beta, p, r, \Delta \phi$. Based on the model- following control in Equation 4, the rudder deflection is calculated as follows.

$$
\delta_{R U D}=\frac{\dot{r}_{m}-\left(\bar{N}_{\beta} \Delta \beta_{m}+\bar{N}_{p} p_{m}+\bar{N}_{r} r_{m}+\bar{N}_{\phi} \Delta \phi_{m}+\bar{N}_{\delta_{H T A}} \delta_{H T A}\right)}{\bar{N}_{\delta_{R U D}}}
$$

where $r_{m}$ is the yaw rate of KFA-m aircraft to be simulated by KFA-i. Finally, for normal load simulation using trailing edge flaperon (TEF) symmetrical, the following form of normal load factor equation is used.

$$
\dot{n}_{z}=n_{z_{\alpha}} \Delta \dot{\alpha}+n_{z_{H T S}} \dot{\delta}_{H T S}+n_{z_{T E F}} \dot{\delta}_{T E F}
$$

where $n_{z_{\alpha}, z_{H T S}, z_{T E F}}$ are normal g-load factor derivatives with respect to $\Delta \alpha, \delta_{H T S}, \delta_{T E F}$. Since

$$
\Delta \dot{\alpha}=q-Z_{\alpha} \Delta \alpha+Z_{\delta_{H T S}} \delta_{H T S}+Z_{\delta_{T E F}} \delta_{T E F}
$$

where $Z_{\alpha, \delta_{H T S}, \delta_{T E F}}$ are normal force derivatives with respect to $\Delta \alpha, \delta_{H T S}, \delta_{T E F}$. Dividing Equation 11 by $n_{z_{\alpha}}$ and applying Equation 12 gives

$$
\frac{\dot{n}_{z}}{n_{z_{\alpha}}}=q-Z_{\alpha} \Delta \alpha+Z_{\delta_{H T S}} \delta_{H T S}+Z_{\delta_{T E F}} \delta_{T E F}+\frac{n_{z_{H T S}}}{n_{z_{\alpha}}} \dot{\delta}_{H T S}+\frac{n_{z_{T E F S}}}{n_{z_{\alpha}}} \dot{\delta}_{T E F}
$$

Using the following relationships,

$$
\frac{n_{z_{H T S}}}{n_{z_{\alpha}}}=\frac{Z_{\delta_{H T S}}}{Z_{\alpha}}, \frac{n_{z_{T E F}}}{n_{z_{\alpha}}}=\frac{Z_{\delta_{T E F}}}{Z_{\alpha}}
$$

the TEF deflection is calculated as follows.

$$
\delta_{T E F}=\frac{\frac{\dot{n}_{z_{m}}}{n_{z_{\alpha_{m}}}}-\left(q_{m}-Z_{\alpha} \Delta \alpha_{m}+\left(Z_{\delta_{H T S}}+s \frac{\left.\left.Z_{\delta_{H T S}}\right) \delta_{\alpha}\right)}{Z_{\delta_{T E F}}+\frac{Z_{\delta_{T E F}}}{Z_{\alpha}} s}\right.\right.}{Z_{\alpha T S}}
$$

where $s$ is an operator of a Laplace transform, and $\dot{n}_{z_{m}}$ is the rate of normal load factor of the KFA-m aircraft and it can be simulated by KFA-i.

\subsection{Feedback Control}

Usually, exact model-following is not achievable with feed-forward control only, and thus the additional feedback control is required. The objective of feedback control is to reduce the model-following errors incurred by feed-forward control. We apply feedback control as follows:

$$
\mathbf{u}_{f b}=\mathbf{K}_{p}\left(\mathbf{x}_{m}-\mathbf{x}_{p}\right)+\mathbf{K}_{I} \int\left(\mathbf{x}_{m}-\mathbf{x}_{p}\right) d t
$$

The proportional and integral gains are designed from the classical PI design technique. The design of feedback control can be performed independently from that of feed-forward control.

\subsection{Switching and Fader Logic}

While model-following control law works when the VSS mode is on, the baseline KFA-i flight control system is used when the VSS mode is not engaged. The system allows the pilot to specify VSS on mode, and both the pilot and the automatic limit-monitoring system may revert to the baseline flight control system upon mission completion or an abnormal condition. Because both the baseline and the model-following control laws are computed continuously during flight, actuator commands from the model-following control law and the baseline flight control law of the KFA-i aircraft should be selected by the switching logic. Moreover, the fader logic is also required to prevent the aircraft from experiencing large transient motion during the mode switching between VSS on and off-modes.

In this paper, the following linear fusing type of fader logic is used for switching to the baseline flight control system $[8,9]$ :

$$
\delta_{\text {FADER }}(t)=\frac{\Delta t}{T} \delta_{B A S E}(t)+\left(1-\frac{\Delta t}{T}\right) \delta_{M F C}(t)
$$

where $\delta_{B A S E}$ and $\delta_{M F C}$ are actuator commands calculated by the baseline flight control law and the model-following control law, respectively, and $T$ is a fading time, and $\Delta t$ is a fading time counter having a value between 0 and $T$.

Figure 2 shows one example of a fading factor, which is 
given by the designed fader. After $2 \mathrm{~s}$, switching from A-signal (dotted line) to B-signal (green line) occurred and the fader output signal (red line) shows the fader effect.

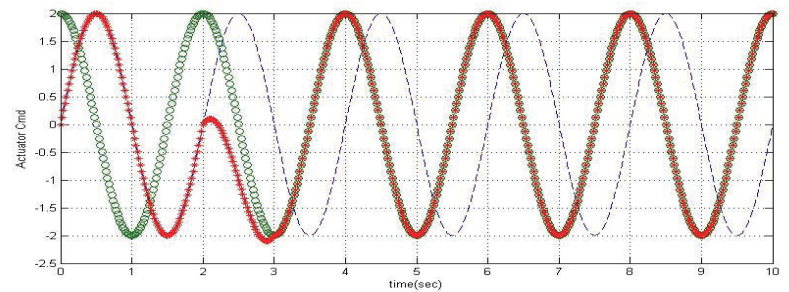

Fig. 2. Example of fading factor of the used fader (dot: A-signal before switching, green: B-signal before switching, red: fader output signal)

\section{Simulation Results}

Simulations were conducted to evaluate the modelfollowing capability and performance of the system for the three flight conditions (LL, LH, HH). The KFA-m aircraft [10] was used as a model to produce the desired aircraft dynamics in KFA-i in-flight simulation aircraft. The resulting system produces a model-following simulation scheme where the KFA-i aircraft recreates KFA-m flight dynamics.

Because it is desirable for the system to show modelfollowing characteristics, pitch/roll/yaw doublets are incorporated into the system. Fig. 3 illustrates the pitch rate doublet simulation results of the $\mathrm{HH}$ condition. In Figure 3 , the pilot's doublet command is applied to the KFA-m's pitch rate control law and the KFA-i pitch rate and g-load responses are matched exactly to the KFA-m's pitch rate and g-load responses by the VSS control law. The deflection of the TEFs and HTs are showed in the Fig. 3.

Figure 4 shows the performance of the VSS control law to simulate the roll rate response of KFA-m. In Fig. 4, the pilot's doublet command is applied to the KFA-m's roll rate control law and the KFA-i roll rate response is matched exactly to the KFA-m's roll rate responses by the VSS control law. The deflection of the HTs are showed in the Fig. 4.
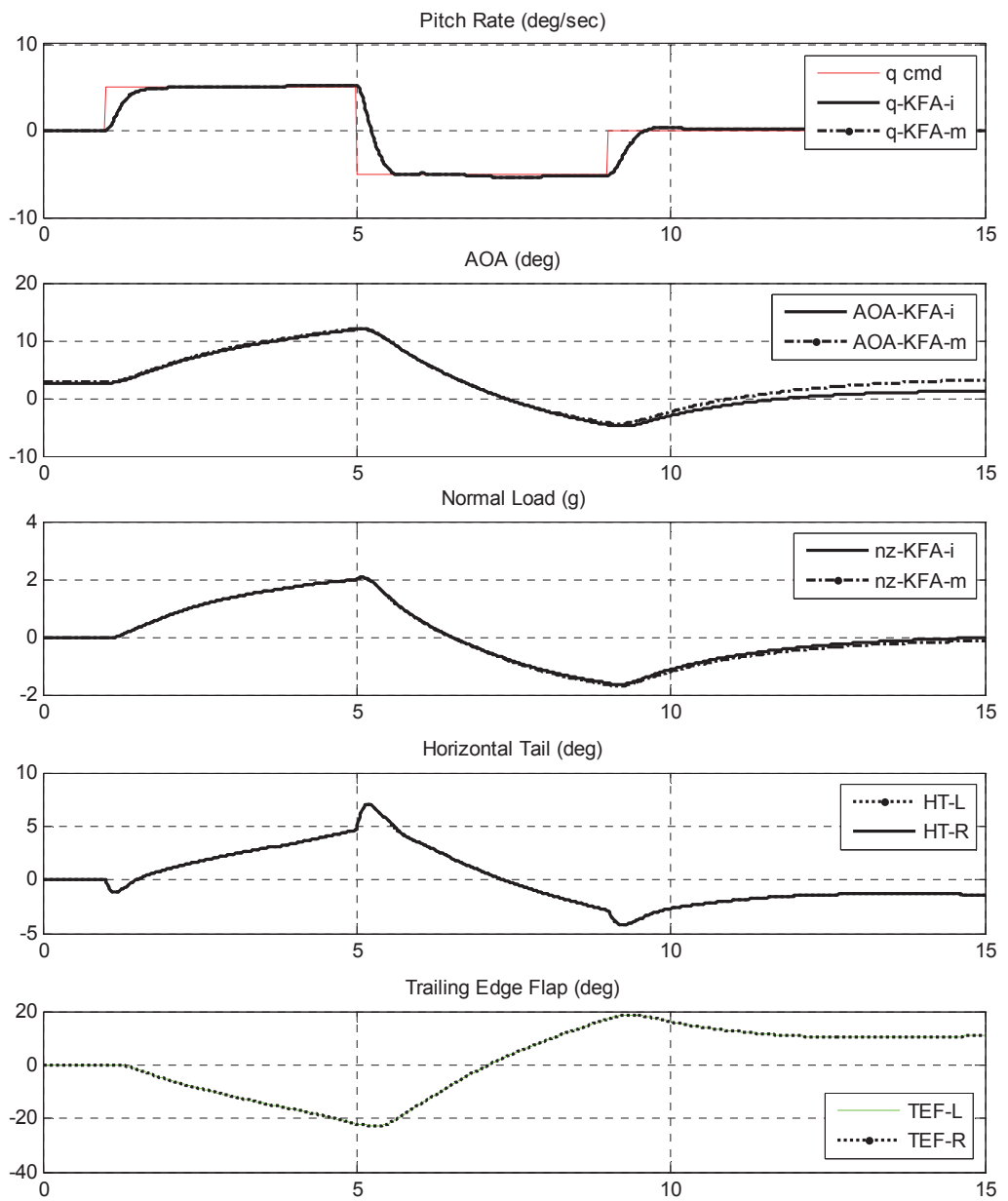

Fig. 3. Pitch doublet response of model A/C and IFS A/C with VSS (HH) 
Figure 5 illustrates the yaw doublet simulation results of the HH condition. In the Fig. 5, the pilot's doublet command is applied to the KFA-m's angle of sideslip (AOS) control law. There are errors between the angle of sideslip responses, as we expect, but we can identify the performance of the VSS control law with the yaw rate response graph. We can also check the KFA-i's rudder deflection to match the yaw rate of the KFA-m in Fig. 5.

The simulation results show that KFA-i follows the responses of pitch/roll/yaw rate and load of KFA-m with high accuracy. From this result, we can check the performance of the KFA-m's flying quality. Analysis of KFA-m longitudinal and lateral-directional handling qualities according to military aircraft's handling qualities requirements [11,12] and with the use of an equivalent system approach reveals that it is preferable to provide some overshoot in pitch rate response to pilot control step input to ensure lower response times and equivalent time delays of KFA-m's load response. Roll motion time constants values are acceptable for $\mathrm{HH}$ flight conditions. Yaw motion characteristics are almost acceptable, but it is desirable to increase damping, up to $\zeta>$ 0.35 , for HH regimes.

\section{Conclusions}

Model-following VSS control laws for KFA-i to simulate the dynamics of the KFA-m aircraft were developed. The system consists of feed-forward and feedback control laws, the KFA-m aircraft dynamic model with control law to be simulated, a baseline flight control of KFA-i, and switching and fader logic.

The feed-forward control was designed by the modelfollowing control method to simulate four parameters - pitch, roll, yaw rates, and load - of the KFA-m aircraft simultaneously. The feedback control laws were designed independently to reduce the model-following errors incurred by feed-forward control alone. In the design of feedback gains, the classical PI control was used. The linear fusing type of fader logic was designed to prevent undesirable transient motion during the mode switching between VSS on- and off-modes. The KFA-m's control law with pitch rate, roll rate and AOS command system was designed to evaluate the VSS control law by simulation.

Computer simulation was performed for VSS validation and analysis. The simulation results showed that KFA-i
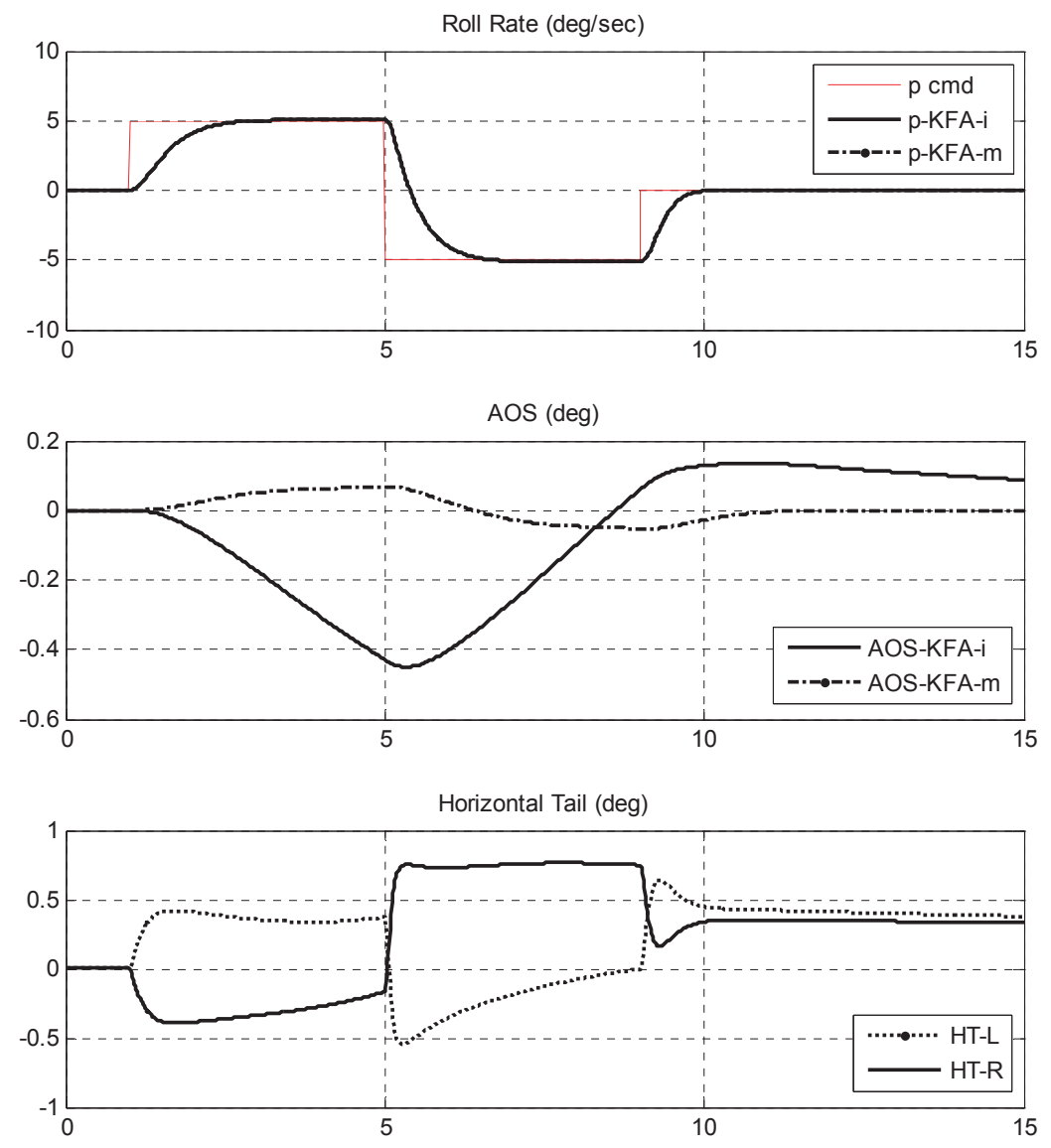

Fig. 4. Roll doublet response of model A/C and IFS A/C with VSS (HH) 

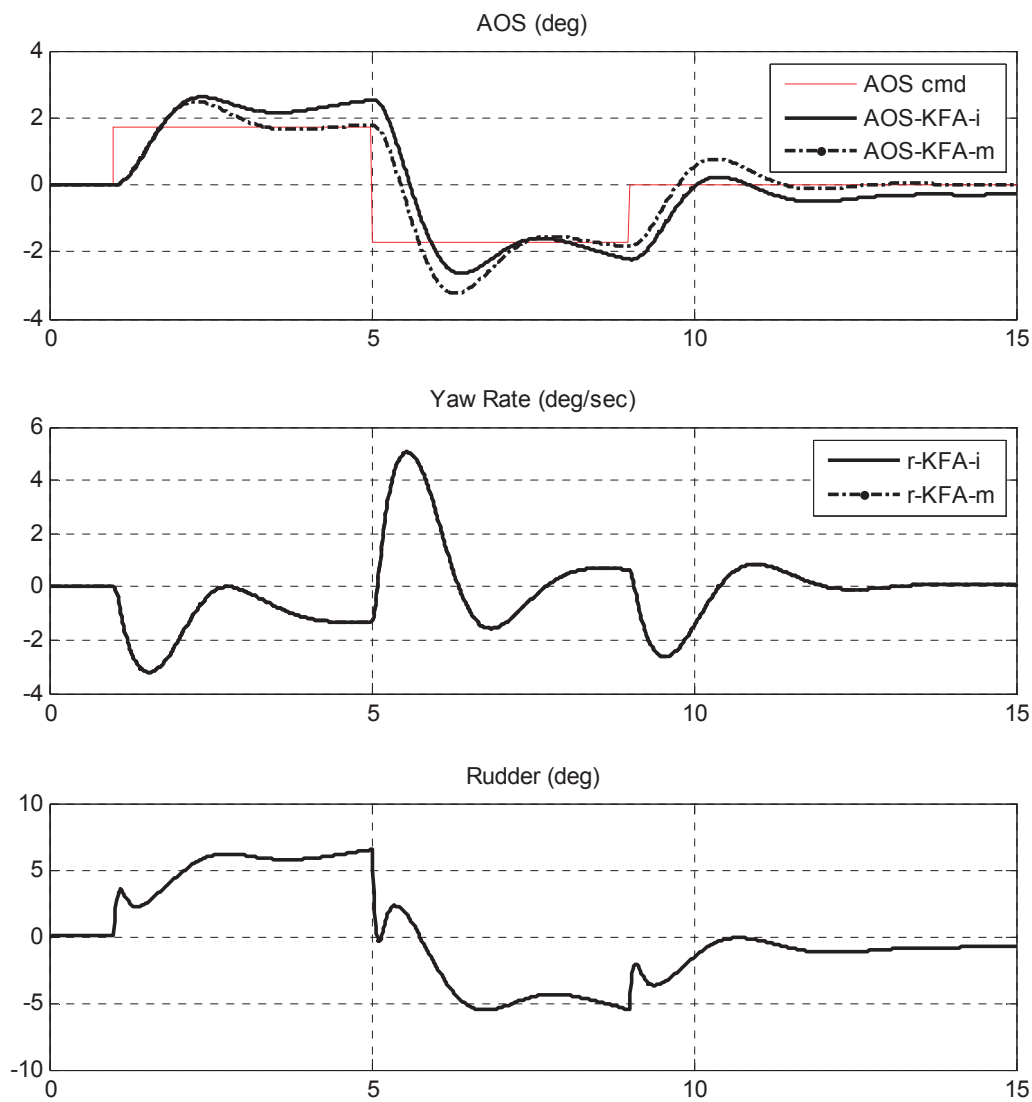

Fig. 5. Yaw doublet response of model A/C and IFS A/C with VSS (HH)

follows the responses of pitch/roll/yaw rate and load of KFA-m with high accuracy and it may be used to simulate dissimilar flight control systems.

\section{Acknowledgements}

This work was supported by the Industrial Strategic Technology Development Program (10040188, Helicopter Fly by Wire Flight Control System Development, according to ADS-33), funded by the Ministry of Knowledge Economy (MKE), Korea.

\section{References}

[1] Grantham W., "Comparison of Flying Qualities Derived From In-Flight and Ground-Based Simulators for a Jet-Transport Airplane for the Approach and Landing Pilot Tasks", NASA Technical Paper 2962, Hampton, VA, USA, 1989.

[2] Pettersson H., Variable Stability Transfer Function Simulation, MS Thesis, Virginia Polytech., USA, 2002.
[3] Carter J., "Production support flight control computers", NASA TM-97-206233, 1987.

[4] Ko JS, Lee HK and Lee JY., "In Flight Simulation for Flight Control Law Evaluation of Fly-by-Wire Aircraft", SimTech 2004 Conference, 2004.

[5] Germann K., T-6A Texan II in-flight simulation and variable stability system design, $\mathrm{PhD}$ Thesis, Mississippi State University, USA, 2006.

[6] Zinober A and Yew M. "Variable Structure ModelFollowing Control of Flight Dynamics", AIAA, 1987, pp. 707712.

[7] Hilbert KB, Lebacqz JV and Hindson W S., "Flight investigation of multi-variable model-following control systems for rotorcraft", AIAA Paper No. 86-9779, AIAA $3^{\text {rd }}$ Flight Test Conference, Las Vegas, Nevada. April 2-4 1986.

[8] Park S and Ko JS., "Design of a Variable Stability Flight Control System”, KSAS Inetrnational Journal, Vol. 9, No.1, 2008, pp. 162-168.

[9] Hespanha J, Liberzon D, Morse A and Bruyne F., "Multiple model adaptive control. Part 2, Switching", Inernational Journal of Robust and Nonlinear Control, Vol. 11, 2001, pp. 479-496. 
Int'I J. of Aeronautical \& Space Sci. 15(4), 412-418 (2014)

[10] Stevens B and Lewis F., Aircraft Control and Simulation. $2^{\text {nd }}$ ed. Wiley, 1992.

[11] Mil-HDBK-1797, Flying Qualities of Piloted Aircraft.
1995.

[12] MIL-F-8785C, Flying Qualities of Piloted Airplanes, 1996. 